\title{
Association Studies Between Type 1 (Insulin-Dependent) Diabetes and 27 Genetic Markers: Lack of Association Between Type 1 Diabetes and Kidd Blood Group
}

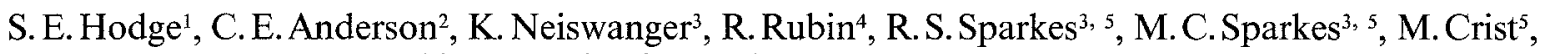 \\ M.A.Spence ${ }^{1,3}$, P.I. Terasaki ${ }^{6}$, D. L. Rimoin ${ }^{4}$, and J. I. Rotter ${ }^{4}$ \\ Departments of ${ }^{1}$ Biomathematics, ${ }^{3}$ Psychiatry, ${ }^{5}$ Medicine and ${ }^{6}$ Surgery, University of California, Los Angeles School of Medicine, \\ ${ }^{2}$ Department of Pediatrics, University of California, Irvine, and \\ ${ }^{4}$ Departments of Pediatrics and Medicine, Harbor-University of California, Los Angeles Medical Center, Torrance, California, USA
}

\begin{abstract}
Summary. One hundred and three unrelated patients with Type 1 (insulin-dependent) diabetes were typed for HLA, properdin factor B (BF), glyoxalase 1 (GLO), Kidd blood group, and 24 other genetic markers. Observed distributions of marker phenotypes among these patients were compared with those expected according to population frequencies, in an attempt to detect associations between Type 1 diabetes and the markers. Strong associations between Type 1 diabetes and both HLA and properdin factor B were confirmed, as was a lack of association between Type 1 diabetes and glyoxalase (GLO). There was an apparent deviation from Hardy-Weinberg equilibrium at the GLO locus, and statistically significant distortions in the distributions of pancreatic amylase (AMY2),
\end{abstract}

galactose-1-phosphate uridyl transferase (GALT), and groupspecific component $(\mathrm{GC})$ among Type 1 diabetes patients, but these results are not significant when corrected for performance of multiple tests. An increase in the Lewis-negative phenotype reported elsewhere was observed here but was not statistically significant. A distortion in the distribution of Kidd types reported elsewhere was not confirmed.

Key words: Type 1 diabetes, Kidd blood group, HLA, glyoxalase, pancreatic amylase, galactose-1-phosphate uridyl transferase, group-specific component, Lewis blood group, disease-marker associations, two-locus inheritance.
Associations with genetic markers may provide clues to the genetics of Type 1 (insulin-dependent) diabetes mellitus. Most studies of associations between Type 1 diabetes and genetic markers have focussed on the chromosome 6 markers: HLA, properdin factor B (BF), and glyoxalase 1. Certain alleles in the HLA system (particularly DR3 and DR4 at the HLA-DR locus), as well as the F1 allele in the BF system, have been shown to be consistently associated with Type 1 diabetes [1-3]. In contrast, no association has been observed with glyoxalase [3]. It should be noted that BF and HLA are tightly linked, whereas glyoxalase is located approximately 10 map units from HLA and BF [4].

Less work has been carried out with genetic markers not on chromosome 6. Lucarelli et al. examined associations between Type 1 diabetes and nine marker systems, with inconclusive results [5]. Vague et al., studying seven blood groups, observed an increase in the Lewis negative (a-b-) red blood cell phenotype, among both Type 1 and Type 2 (non-insulin-dependent) diabetic patients [6]. More recently, we reported possible evidence for genetic linkage between Type 1 diabetes and the Kidd blood group locus [7]. Kidd is thought to be located on chromosome 2 [8]. Subsequently, Barbosa et al. have de- scribed a distortion in the Kidd distribution among a subgroup of Type 1 diabetics, classified by HLA-DR type [9].

\section{Methods}

We looked for possible associations between Type 1 diabetes and 27 genetic markers in a sample of 103 unrelated individuals. The study was divided into three parts: chromosome 6 markers, Kidd blood group, and other markers. Standard gene frequencies, based on Mourant et al. [10], Harris and Hopkinson [11], and Race and Sanger [12], in conjunction with Hardy-Weinberg equilibrium, were used to compute all expected genotype frequencies, except for HLA frequencies, which were taken from the 1980 International Histocompatibility Testing Workshop [13].

The sample of 103 individuals was created from a data set of simplex and multiplex Type 1 diabetic families by selecting the oldest affected family member with complete HLA typing information. The full data set consisted of Type 1 diabetic patients and their families, ascertained through clinics and private diabetologists in the Los Angeles area. Affected persons were defined as being ketosis-prone and having acute onset of insulin dependence before 40 years of age. Of 99 patients with known age of onset, 53 were diagnosed before the age of 10 years, 34 between 10 and 19 years, and 12 at 20 years or older. There were 56 males and 47 females. Forty of the families were sim plex, and the remainder had two or more affected members. All were Caucasoid, with nine of Hispanic background. Complete details are 
Table 1. Observed distribution of HLA-DR and Kidd genotypes among 103 unrelated patients

\begin{tabular}{|c|c|c|c|c|c|c|c|}
\hline \multirow[b]{2}{*}{ Row } & \multirow[b]{2}{*}{ HLA-DR } & \multicolumn{5}{|l|}{ Kidd } & \multirow[b]{2}{*}{$\begin{array}{l}\text { Total } \\
\text { DR }\end{array}$} \\
\hline & & a-positive & aa & $a b$ & $\mathrm{bb}$ & $\begin{array}{l}\text { Not } \\
\text { typed }\end{array}$ & \\
\hline 1 & $3 / 3$ & $0(0)$ & $1(1)$ & $0 \quad(0)$ & $1(1)$ & $1(0)$ & 3 (2) \\
\hline 2 & $3 / X$ & $6(0)$ & $2(2)$ & 3 (3) & $2(0)$ & $2(0)$ & $15 \quad(5)$ \\
\hline 3 & $3 / ?$ & $0(0)$ & $0(0)$ & $0 \quad(0)$ & $0(0)$ & $0(0)$ & $\begin{array}{ll}0 & (0)\end{array}$ \\
\hline 4 & $4 / 4$ & $0(0)$ & $0(0)$ & $0 \quad(0)$ & $2(1)$ & $0(0)$ & $2(1)$ \\
\hline 5 & $4 / X$ & $4(0)$ & $2(1)$ & $10(10)$ & $6(2)$ & $2(0)$ & $24(13)$ \\
\hline 6 & $4 / ?$ & $7(0)$ & $0(0)$ & $3(2)$ & $0(0)$ & $2(0)$ & $12(2)$ \\
\hline 7 & $3 / 4$ & $8(0)$ & $4(4)$ & $10(10)$ & $5(3)$ & $7(0)$ & $34(17)$ \\
\hline 8 & $\mathrm{X} / \mathrm{X}$ & $2(0)$ & $0(0)$ & $2(1)$ & $1(0)$ & $2(0)$ & 7 (1) \\
\hline 9 & Not typed & $3(0)$ & $1(1)$ & $0 \quad(0)$ & $2(1)$ & $0(0)$ & 6 (2) \\
\hline Tot & idd & $30(0)$ & $10(9)$ & $28(26)$ & $19(8)$ & $16(0)$ & $103(43)$ \\
\hline
\end{tabular}

Numbers in parentheses indicate observations among the 43 individuals typed for both Kidd $a$ and $b$ alleles.

a-positive = typed for Kidd $a$ only (see text); X=not DR3 or DR4; $?=$ homozygosity ambiguous

given by Anderson et al. [14]. The HLA-DR data have also been described elsewhere [15]. If no affected person had complete HLA typing, the oldest affected individual was selected.

Each subject was typed for up to 27 red blood cell antigens, red blood cell enzymes, and serum protein markers, using standard laboratory techniques. The markers were: ABO blood group, acid phosphatase-1 (ACP1), adenosine deaminase (ADA), adenylate kinase-1 (AK1), $\alpha$-amylase (pancreatic) (AMY2), properdin factor B (BF), cholinesterase-2 (CHE2), esterase D (ESD), Duffy blood group (Fy), galactose-1-phosphate uridyl transferase (GALT), group-specific protein (GC), glyoxalase 1 (GLO), glutamic-oxaloacetic transaminase (soluble) (GOTS), glutamic-pyruvic transminase (GPT), HLA, haptoglobin (HPA), Kidd blood group (Jk), Kell blood group (K), Lewis blood group (Le), MNSs blood group, P blood group, phosphogluconate dehydrogenase (PGD), phosphoglucomutase-1 and -2 (PGM1 and PGM2), phosphoglycollate phosphatase (PGP), Rhesus blood group (Rh), and transferrin (TF). Gene symbols are those standardized by the Human Gene Mapping Conference [16]. (Not every individual was typed for every marker; e. g., some individuals were typed only for presence versus absence of the Kidd $a$ allele, due to a temporary lack of Kidd anti- $b$ antisera).

In the BF system, the proportion of patients with the $\mathrm{F} 1$ allele was compared with the expected. In the GLO system, the observed frequencies of the two alleles, 1 and 2, as determined by gene counting [17], were compared with the expected; then the genotype frequencies were compared with those expected under Hardy-Weinberg equilibrium. In the HLA system, the distribution of alleles DR3 and DR4 at the DR locus was compared with expected numbers.

The Kidd blood group was examined in three ways: (a) The distribution of Kidd genotypes among the Type 1 diabetic patients, treated as a group, was compared with expected population frequencies. (b) Following the approach of Barbosa et al. [9], patients were divided into high- and low-risk groups, depending on whether they had two doses of the 'high-risk' HLA-DR alleles DR3 and DR4 or fewer than two doses. Thus, high-risk patients had genotypes DR3/3,3/4, or $4 / 4$, and low-risk patients had genotypes $D R 3 / X, 4 / X$, or $X / X$, where ' $X$ ' indicates any $D R$ allele that is neither DR3 nor DR4. The two groups were then examined for differential Kidd associations. Whether an individual who typed for only DR3 or DR4 was in fact homozygous was determined by genotyping the family. If homozygosity was ambiguous, the individual was not included in this analysis. (c) Several additional relationships between HLA and Kidd types were examined among those patients who had at least one dose of DR3 and/ or DR4 and who had complete typing for both HLA and Kidd.

For the remaining genetic markers, observed phenotype frequencies were compared with expected frequencies.

\section{Statistical Methods}

Depending on sample size, the $\chi^{2}$ test, the normal approximation to the binomial, or Fisher's exact test was used for statistical comparisons.

\section{Results}

\section{Chromosome 6 Markers}

Properdin factor $B$ : Of 86 patients with BF typing, 13 $(15.1 \%)$ had the F1 allele (two were F1F, 11 were F1S). The expected proportion of $\mathrm{F} 1$ carriers in the general population is $0.9 \%$, based on an F1 allele frequency of 0.0045 . This deviation from expected is highly significant $(p=$ negligible, based on the normal approximation to the binomial distribution).

Glyoxalase ( $G L O$ ): Ninety-nine patients were typed for GLO. The observed genotype distribution was:

$\begin{array}{lrrr}\text { GLO genotype } & 1-1 & 2-1 & 2-2 \\ \text { Number observed } & 28 & 38 & 33\end{array}$

The observed frequency of allele GLO-1, 0.47, did not differ significantly from the expected frequency of 0.45 $\left(\chi^{2}=0.49,1\right.$ d.f., $\left.0.50>p>0.30\right)$. However, the above genotype distribution does exhibit a deficit of 2-1 heterozygotes compared with the number expected under Hardy-Weinberg equilibrium, using the observed gene frequency of 0.47 (goodness-of-fit $\chi^{2}=5.26,1$ d.f., $p<$ $0.05)$.

HLA: The extreme right column of Table 1, 'Total DR', shows the complete breakdown of HLA genotypes at the DR locus. Of 97 patients with DR typing, 52 (54\%) typed for DR3 and $72(74 \%)$ typed for DR4, of whom 34 $(35 \%)$ were DR3/4. Only seven $(7 \%)$ had neither DR3 nor DR4. Looking at the 85 patients in whom DR3 or DR4 homozygosity could be determined unambiguously, the genotypes were distributed as follows:

$\begin{array}{llllll}3 / 3 & 3 / \mathrm{X} & 4 / 4 & 4 / \mathrm{X} & 3 / 4 & \mathrm{X} / \mathrm{X} \\ 3 & 15 & 2 & 24 & 34 & 7\end{array}$

As before, ' $\mathrm{X}$ ' indicates any DR allele that is neither DR3 nor DR4. Allele frequencies estimated by gene counting are: DR3 $=0.324, \quad$ DR $4=0.365$ and $\mathrm{DRX}=0.312$. These differ significantly from population frequencies of 0.118 for DR3 and 0.148 for DR4 [13] (goodness-of-fit $\chi^{2}=156.5,2$ d.f., $p=$ negligible). Moreover, even using these gene frequencies estimated from the data set, the genotype distribution differs significantly from that expected under Hardy-Weinberg equilibrium (goodness-of-fit $\chi^{2}=22.8,3$ d.f., $p<$ 0.0001 ). The greatest deviation occurs among $3 / 4$ heterozygotes, where the expected number is 20.1 , and that observed is 34 . 
Table 2. Distribution of Kidd blood group genotypes among Type 1 diabetic patients classified as 'high'- and 'low-risk' according to HLA-DR genotypes

\begin{tabular}{lcccc}
\hline & \multicolumn{2}{c}{ 'High-risk' } & & 'Low-risk' \\
\cline { 2 - 3 } Kidd genotype & DR 3/3,4/4 or 3/4 & & DR 3/X, 4/X or X/X & Total \\
\hline aa & 5 & 4 & 9 \\
ab & 10 & 15 & 25 \\
bb & 8 & 28 & 17 \\
\hline Total & 23 & 9 & 51 \\
\hline
\end{tabular}

$\chi^{2}=0.66(2$ d.f., $0.80>p>0.70)$

Table 3. Distribution of homozygotes and heterozygotes at the HLA and Kidd loci

\begin{tabular}{llll}
\hline \multirow{2}{*}{ Kidd } & \multicolumn{2}{l}{ HLA-DR } & \\
\cline { 2 - 4 } & Homozygous & Heterozygous & \\
& $3 / 3,4 / 4$ & $3 / \mathrm{X}, 4 / \mathrm{X}, 3 / 4$ & \\
\hline Homozygous aa, bb & 3 & 12 & 15 \\
Heterozygous ab & 0 & 23 & 23 \\
\hline & 3 & 35 & 38 \\
\hline
\end{tabular}

Fisher's exact test: $2 p=0.11$

Table 4. Sample sizes and $p$ values for association tests between Type 1 diabetes and 23 genetic markers

\begin{tabular}{lll}
\hline Marker locus & Number of patients & $p$ value $^{\mathrm{a}}$ \\
\hline ABO & 87 & $p>0.9$ \\
ACP1 & 87 & $0.7>p>0.5$ \\
ADA & 87 & $0.9>p>0.8$ \\
AK1 & 87 & $0.7>p>0.5$ \\
AMY2 & 85 & $0.02>p>0.01$ \\
CHE2 & 87 & $0.2>p>0.1$ \\
ESD & 87 & $0.5>p>0.3$ \\
Fy & 87 & $0.5>p>0.3$ \\
GALT & 89 & $0.01>p>0.005$ \\
GC & 75 & $0.05>p>0.02$ \\
GOTS & 15 & $p=0.94$ \\
GPT & 86 & $0.8>p>0.7$ \\
HPA & 87 & $0.3>p>0.2$ \\
K & 87 & $p>0.9$ \\
Le & 60 & $p=0.17$ \\
MNSs & 86 & $0.7>p>0.5$ \\
P & 87 & $0.1>p>0.05$ \\
PGD & 87 & $0.5>p>0.3$ \\
PGM1 & 87 & $0.5>p>0.3$ \\
PGM2 & 15 & $p=0.97$ \\
PGP & 72 & $0.5>p>0.3$ \\
Rh & 88 & $0.8>p>0.7$ \\
TF & 82 & $p=0.37$ \\
\hline
\end{tabular}

a Not corrected for multiple tests. Abbreviations are defined under Methods

\section{Kidd Blood Group}

Of the 87 patients typed for Kidd (the "complete sample'), 43 (the 'reduced sample') were typed for both the Kidd $a$ and $b$ alleles, whereas 44 were typed only for presence versus absence of $a$. Table 1 gives the Kidd distribution for all 87 individuals and, in parentheses, for the reduced sample. Results from the three analyses of the Kidd blood group (see Methods) will now be given in turn.

(a) Expected genotype frequencies were calculated according to Hardy-Weinberg equilibrium, using 0.51 for the frequency of the Kidd $a$ allele $[10,12]$. Neither the observed distribution of $68 a$-positive, $19 a$-negative in the complete sample nor the distribution of all three genotypes, $a a, a b$, and $b b$ in the reduced sample $\left(\chi^{2}=1.89,2\right.$ d.f., $\left.0.50>p>0.30\right)$ was significantly distorted.

(b) Among 'low-risk' patients in the reduced sample, 3 were $a a, 14, a b$, and $2, b b$ (rows 2, 5 and 8, Table 1 ). This observed frequency of the Kidd $b$ allele, 0.47 , does not differ significantly from, and is in fact slightly less than, the expected frequency of 0.49 . Comparing highversus low-risk patients directly, in the complete sample, does not support any increase in Kidd $b$ among low-risk patients, nor is any difference observed between low- and high-risk patients (Table 2).

(c) Thirty-eight patients had both complete Kidd typing and complete HLA typing (with no ambiguity as to homozygosity) and at least one DR3 or DR4 allele (rows 1, 2, 4, 5, and 7 and columns 2, 3, and 4, Table 1, data in parentheses). There was no difference in Kidd types between DR3/4 and non-3/4 patients $\left(\chi^{2}=0.01,2\right.$ d.f.). Alleles at the two loci were distributed independently $\left(\chi^{2}=0.92,2\right.$ d.f.). The 38 patients exhibited a slight but non-significant increase in double heterozygotes (Fisher's exact test, two-tailed $p=0.11$; Table 3 ).

\section{Other Markers}

Table 4 gives the number of people typed and the statistical $p$ value for each of the 23 remaining markers. (Detailed marker distributions are available upon request.) All $p$ values are based on a goodness-of-fit $\chi^{2}$ test, except for four markers (GOTS, Lewis, PGM2 and TF). For these markers, the exact binomial $p$ values were computed, since the expected numbers were too small for the $\chi^{2}$ test. Three markers deviated significantly from expected proportions when considered alone: AMY2, GALT and GC. AMY2 exhibited an excess of the AA genotype; GALT exhibited an excess of ND; and GC exhibited an excess of 2-1 and a deficit of 1-1. However, multiplying the $p$ values by the total number of tests performed, to allow for multiple tests [18], removes this apparent statistical significance. Table 5 shows the distributions of these three markers.

The Lewis negative phenotype, a-b-, was slightly increased in our sample (expected 3.7, observed 6), but the increase was not statistically signficant $(p=0.17)$.

\section{Discussion}

The three goals of this study were: (1) to confirm the HLA and BF associations and the lack of GLO associa- 
Table 5. Marker distributions for three markers yielding statistically significant results in Table 4

\begin{tabular}{lllll}
\hline & Genotype & & AA & AA, BB \\
\hline AMY2 & Observed & & 83 & 2 \\
& Expected & & 76.1 & 8.9 \\
\hline \multirow{2}{*}{ GALT } & Genotype & NN & ND & $\begin{array}{l}\text { NL, NG } \\
\text { and other }\end{array}$ \\
& & & & 3 \\
& Observed & 72 & 14 & 5.9 \\
\hline GC & Expected & 76.5 & 6.6 & $2-2$ \\
& Genotype & $1-1$ & $1-2$ & 6 \\
& Observed & 28 & 41 & 6.1 \\
\hline
\end{tabular}

tion observed in other Type 1 diabetic data sets; (2) to determine if there were any association between Type 1 diabetes and the Kidd blood group, either among Type 1 diabetic patients as a whole or in those classified by HLA type; and (3) to determine whether any other markers (not on chromosome 6 or Kidd) were associated with Type 1 diabetes.

The strong associations of Type 1 diabetes with the HLA alleles DR3 and DR4 and with the BF allele F1 agree with those reported elsewhere [1-3], as does the lack of allelic association between Type 1 diabetes and GLO. Thus, our data set is compatible in this regard with others reported in the literature. In addition, even when the allele frequencies estimated from the data set are used, the HLA genotype distribution deviates significantly from Hardy-Weinberg equilibrium, with a large excess of DR3/4 heterozygotes.

The Kidd marker was analyzed in three ways: (a) there was no deviation from expected Kidd population frequencies, in either the reduced sample of 43 individuals with complete typing or the full sample of 87 individuals; (b) genotype frequencies in high- and low-risk patients, considered as separate groups, did not differ from expected population frequencies, nor did the two groups differ from each other; (c) there were no significant distortions in the relationships of HLA and Kidd distributions.

The four statistically signficant results in this study, apart from the anticipated deviations at the HLA and BF loci, were: an excess of the AA type at the pancreatic amylase locus on chromosome 1, a deficit of 2-1 heterozygotes at the GLO locus on chromosome 6 , an excess of the ND type at the GALT locus on chromosome 9, and an excess of the 2 allele at the group-specific component locus on chromosome 4 . Given the large number of tests performed, these four results should not be considered as proven unless confirmed elsewhere.

We also observed a non-significant increase in the Lewis negative phenotype, in the same direction as reported by Vague et al. [6], and a non-significant increase in the number of double HLA-Kidd heterozygotes.
We failed to confirm here two results reported elsewhere - an excess of the Kidd $b$ allele among low-risk patients [9] and an excess of the Lewis-negative a-bphenotype among all patients [6]. It is therefore appropriate to consider whether our sample sizes were adequate to detect the effects if they did exist, i.e., to examine the power of the tests.

For Kidd, the power of the test is $56 \%$ : Let " $F$ " denote the frequency of the $b$ allele among low-risk patients. The null hypothesis is $\mathrm{H}_{0}: \mathrm{F}=0.49$, based on the expected population frequency; the alternative hypothesis is $\mathrm{H}_{1}: \mathrm{F}=0.586$, based on the frequency observed by Barbosa et al. [9]. Thus for our sample of 19 individuals ( $=38$ alleles) and our predetermined significance level of 0.05 , the probability of correctly rejecting $\mathrm{H}_{1}$ when $\mathrm{H}$ is true is $56 \%$. Calculations are based on the Normal approximation to the binomial distribution, using one-sided probabilities [19]. It is worth noting that if the true frequency $\mathrm{F}$ was 0.586 , then the probability of observing, as we did, a frequency as low as 0.47 among our 19 patients was only 0.097 .

For Lewis, let " $F$ " denote the frequency of the Lewis-negative phenotype among Type 1 diabetic patients, with $\mathrm{H}_{0}: \mathrm{F}=0.062$ versus $\mathrm{H}_{1}: \mathrm{F}=0.29$ (based on Vague et al. [6]). For the sample of 60 patients with Lewis typing, the power of this test is $99.9 \%$.

Except for GOTS and PGM2, all other markers were examined in samples of at least 72 patients (Table 4). Therefore, the power of the remaining tests was probably reasonably good. We cannot calculate exact power levels without specifying alternative hypotheses.

We used published gene frequencies for our comparisons because of the difficulty in determining appropriate controls. Results were unchanged when the analyses were repeated without the nine Hispanic families.

Evidence from population and family studies makes it clear that genes in or near the HLA complex influence genetic susceptibility to Type 1 diabetes. The significance of associations with genes on chromosome 6 , and in particular of the excess of DR $3 / 4$ heterozygotes Type 1 diabetes, has been discussed at length elsewhere $[15,28-30]$. What is unclear is whether other in genetic loci are also involved. A number of tentative and sometimes conflicting reports have appeared regarding possible gene marker associations with Type 1 diabetes. These include the Lewis blood group [6], acetylator [20-22], the Kidd blood group [9], the light and heavy chain immunoglobulin loci [23-25], and, in this report, associations with AMY2, GALT and GC. The picture is further complicated by ambiguous results from linkage studies between Type 1 diabetes and the Kidd blood group [7, 26, 27]. Clearly, additional studies will be needed to answer definitively whether another locus (or loci), not on chromosome 6, is involved in the genetics of Type 1 diabetes.

Acknowledgements. Support for this research was provided by grant AM-25834 from the National Institutes of Health, a grant from the 
Kroc Foundation, and the UCLA Mental Retardation Research Center (NIH grant HD-04612). Computer assistance was obtained from the UCLA Office of Academic Computing and from the UCLA Human Genetics Laboratory's PDP 11/44.

\section{References}

1. Svejgaard A, Platz P, Ryder LP (1980) Insulin-dependent diabetes mellitus (joint report). In: Terasaki PI (ed) Histocompatibility testing 1980. UCLA Tissue Typing Laboratory, Los Angeles, pp 638-656

2. Raum D, Alper CA, Stein R, Gabbay KH (1979) Genetic marker for insulin-dependent diabetes mellitus. Lancet 1: 1208-1210

3. Kirk RL, Theophilus J, Whitehouse S, Court J, Zimmet P (1979) Genetic susceptibility to diabetes mellitus: the distribution of properdin factor $\mathrm{B}(\mathrm{Bf})$ and glyoxalase (GLO) phenotypes. Diabetes 28: 949-951

4. Weitkamp LR, Lamm LU (1982) Report of the committee on the genetic constitution of chromosome 6. Cytogen Cell Genet 32: 130-143

5. Lucarelli P, Scacchi R, Corbo RM, Palmarino R, Orsini M, Campea L, Carapella E, Pascone R (1978) Genetic polymorphisms in juvenile-onset diabetes. Hum Hered 28: 89-95

6. Vague Ph, Melis C, Mercier P, Vialettes B, Lassmann V (1978) The increased frequency of the Lewis negative blood group in a diabetic population. Diabetologia 15:33-36

7. Hodge SE, Anderson CE, Neiswanger K, Field LL, Spence MA, Sparkes RS, Sparkes MC, Crist M, Terasaki PI, Rimoin DL, Rotter JI (1981) Close genetic linkage between diabetes mellitus and Kidd blood group. Lancet 2: 893-895

8. McAlpine PJ, Bootsma D (1982) Report of the Committee on the Genetic Constitution of Chromosomes 2, 3, 4, and 5. Cytogenet Cell Genet 32: 121-129

9. Barbosa J, Rich S, Dunsworth T, Swanson J (1982) Linkage disequilibrium between insulin-dependent diabetes and the Kidd blood group Jk ${ }^{\mathrm{b}}$ allele. J Clin Endocrinol Metab 55: 193-195

10. Mourant AE, Kopec AC, Domaniewska-Sobczak K (1976) The distribution of the human blood groups and other polymorphisms, 2nd edn. Oxford University Press, London

11. Harris H, Hopkinson DA (1966) Handbook of enzyme electrophoresis in human genetics. North-Holland, Amsterdam

12. Race RR, Sanger R (1975) Blood groups in man, 6th edn. Blackwell Scientific, Oxford

13. Baur MP, Danilovs JA (1980) Population analysis of HLA-A, B, $\mathrm{C}, \mathrm{DR}$ and other genetic markers (joint report). In: Terasaki PI (ed) Histocompatibility testing 1980. UCLA Tissue Typing Laboratory, Los Angeles, pp 638-656

14. Anderson CE, Hodge SE, Rubin R, Rotter JI, Terasaki PI, Irvine WJ, Rimoin DL (1983) A search for heterogeneity in insulin dependent diabetes mellitus: HLA and autoimmune studies in simplex, multiplex and multigenerational families. Metabolism 32: 471-477

15. Rotter JI, Anderson CE, Rubin R, Congleton JE, Terasaki PI, Rimoin DL (1983) An HLA genotypic study of insulin dependent diabetes: the excess of DR3/DR4 heterozygotes allows rejection of the recessive hypothesis. Diabetes 32: 169-174

16. Shows TB, McAlpine PJ (1982) The 1981 catalogue of assigned human genetic markers and report of the nomenclature committee. Cytogenet Cell Genet 32: 221-245

17. Emery AEH (1976) Methodology in medical genetics. Churchill Livingstone, Edinburgh, pp 5-6

18. Svejgaard A, Jersild C, Staub Nielsen L, Bodmer WF (1974) HL-A antigens and disease: statistical and genetical considerations. Tissue Antigens 4: 95-105

19. Kendall MG, Stuart A (1973) The advanced theory of statistics, vol 2, 3rd edn. Hafner, New York, pp 187-188

20. McLaren EH, Borden AC, Moorhead PF (1977) Acetylator phenotype in diabetic neuropathy. Br Med J 2: 291-293

21. Burrows AW, Hockaday TDR, Mann JI, Taylor JG (1978) Diabetic dimorphism according to acetylator status. Br Med J 1: 208-210

22. Bodansky HJ, Drury PL, Cudworth AG, Evans DA (1981) Acetylator phenotypes and Type 1 (insulin-dependent) diabetics with microvascular disease. Diabetes 30:907-910

23. Nakao Y, Matsumoto H, Miyazaki T, Mizuno N, Arima N, Wakisaka A, Okimoto K, Akazawa Y, Tsuji K, Fujita T (1981) IgG heavy-chain $(\mathrm{Gm})$ allotypes and immune response to insulin in insulin-requiring diabetes mellitus. New Engl J Med 304: 407-409

24. Schanfield MS, Brown R, Serjeantson S, Kirk RL (1981) Susceptibility to juvenile onset diabetes in families: DR, Gm, Km and gender. Am J Hum Genet 33: 133A

25. Field LL, Anderson CE, Neiswanger K, Sparkes RS, Rimoin DL (1982) Interaction of HLA and immunoglobulin (Gm and $\mathrm{Km}$ ) antigens in insulin-dependent diabetes mellitus. Am J Hum Genet 34: $181 \mathrm{~A}$

26. Dunsworth TS, Rich SS, Swanson J, Barbosa J (1982) No evidence for linkage between diabetes and the Kidd marker. Diabetes 31: 991-993

27. Hodge SE, Anderson CE, Neiswanger K, Sparkes RS, Rimoin DL (1983) The search for heterogeneity in insulin-dependent diabetes: linkage studies, two-locus models, and genetic heterogeneity. Am J Hum Genet (in press)

28. Hodge SE, Rotter JI, Lange KL (1980) A three-allele model for heterogeneity of juvenile onset insulin-dependent diabetes. Ann Hum Genet 43: 399-412

29. Curie-Cohen M (1981) HLA antigens and susceptibility to juvenile diabetes: do additive relative risks imply genetic heterogeneity? Tissue Antigens 17: 136-148

30. Dunsworth TS, Rich SS, Morton NE, Barbosa J (1982) Heterogeneity of insulin-dependent diabetes - new evidence. Clin Genet $21: 233-236$

Received: 20 December 1982

and in revised form: 5 May 1983

Dr. Susan E. Hodge

Mental Retardation Research Center

760 Westwood Plaza

UCLA School of Medicine

Los Angeles, CA 90024, USA 